BERNHARD KLAUSNITZER $^{\text {a }}$ - HASSAN GHAHARI ${ }^{\text {b }}$

\title{
AN ANNOTATED CHECKLIST OF SCIRTOIDEA (COLEOPTERA) OF IRAN
}

\author{
a Senckenberg Deutsches Entomologisches Institut, Lannerstraße 5, 01219 Dresden, Germany $\left(220^{\text {th }}\right.$ contribution to the \\ knowledge of Scirtidae) \\ ${ }^{\mathrm{b}}$ Department of Plant Protection, Yadegar-e-Imam Khomeini (RAH) Shahre Rey Branch, Islamic Azad University, \\ Tehran, Iran \\ Corresponding Author: Hassan Ghahari; hghahari@yahoo.com
}

Klausnitzer B., Ghahari H. - An annotated checklist of Scirtoidea (Coleoptera) of Iran.

The fauna of Iranian Scirtoidea (Coleoptera) is summarized in this paper. In total, three species of Clambidae (within the genus Clambus Fischer von Waldheim, 1821), one species of Eucinetidae (within single genus Bisaya Reitter, 1884), and 23 species of Scirtidae (within the genera Contacyphon Gozis, 1886, Elodes Latreille, 1797, Hydrocyphon Redtenbacher, 1858, Microcara C. G. Thomson, 1859, and Scirtes Illiger, 1807) are listed as members of the Iranian fauna.

Key Words: Elateriformia, Clambidae, Eucinetidae, Scirtidae, distribution, new record, Iran.

\section{INTRODUCTION}

The superfamily Scirtoidea Fleming, 1821 (former Eucinetoidea) includes four families, Clambidae Fischer von Waldheim, 1821 (incl. Calyptomeridae), Decliniidae Nikitsky, Lawrence, Kirejtshuk \& Gratshev, 1994, Eucinetidae Lacordaire, 1857, and Scirtidae Fleming, 1821 (= Cyphonidae, Helodidae, Elodidae) (LAwrence \& NEwTON, 1995). Except for Decliniidae, the other families are represented in the fauna of Iran.

The family Clambidae (Minute Beetles) comprises about 150 species in five genera worldwide (LESCHEN, 2016). They vary in size from $0.7-2.0 \mathrm{~mm}$, are convex and contractile, capable of deflecting their head and pronotum into a seedlike form (Young, 2002b). Metacoxal plates enlarged and legs partly concealed; tarsi 4-4-4 or rarely 3-3-3; abdomen with five or six free ventrites; functional spiracles present on segment VIII (LESCHEN, 2016). They are found throughout the world on all continents (except for the Antarctic) and on many scattered and isolated islands in the Pacific and Indian Oceans (Young, 2002b; MAJKA \& LANGOR, 2009). Most adults are collected from decaying vegetation, leaf litter and rotten wood, and sometimes flying at dusk (Young, 2002b). Most species are known to feed on microfungi or spores and hyphae on larger fruiting bodies of Ascomycetes, Myxomycetes, Basidiomycetes and Zygomycetes (Lawrence \& Newton, 1980; Wheeler \& HoebeKe, 1984; Lawrence, 1991).

The family Eucinetidae (Plate-thigh Beetles) comprises eight genera and more than 40 valid species worldwide (VIT, 1999, 2000). Distinguishing features of adult Eucinetidae include a streamlined elliptical body and the ability to jump using their modified hind legs (KryZhanovskiJ, 1989; Mifsud, 2001; MaJKa, 2010; WeBSTER et al., 2012). The development of the two stages, the adults and the larvae, is strongly connected with the mould fungi, as the adults could be found also under bark or in wood of decayed trees (NIKITSKY, 1989; VIT, 2000). Little is known about the biology of these beetles, but most records result from collections made in leaf litter. In some cases it has been asserted that these insects feed on spores of slime moulds [Myxomycetes (=Mycetozoa)] or on the fruiting bodies of basidiomycete fungi (Agaricaceae, Bolitaceae, and Coniphoraceae) (WheELER \& HoEBeKe, 1984; Young, 2002a; GuÉORGUIEV, 2004).

To date over than 1800 species of the family Scirtidae (Marsh Beetles) have been described. However, a total of at least 3000 species in all zoogeographic regions can be expected (KLAUSNITZER, 2016a, 2017). Important features for the specific identification of the adults (when examined externally) are: length ratios and form of the $1^{\text {st }}-4^{\text {th }}$ antennal segment, punctures of head and pronotum, shape of the pronotum, shape and hairiness of the $7^{\text {th }}$ sternite, colouring (in some genera). Features which require special preparation: shape and fine structure of the $8^{\text {th }}$ and $9^{\text {th }}$ tergite and sternite, tegmen, penis (male), sclerites in bursa (prehensor and bursal sclerite) and the $8^{\text {th }}$ sternite (female). For most species a genital preparation is necessary. Larvae are generally aquatic and frequent stagnant and flowing waters such as streams, lakes, ponds, swamps and sphagnum bogs (Young, 2002c; WeBster et al., 2012), tolerating significant fluctuations of the water level, where they gather algae and other small particles from liquid substrates (MARINONI et al., 2001; Young, 2002c). Sometimes larvae of some species develop in groundwater as deep as 10 meters (KLAUSNITZER \& POSPISIL, 1991). Adults of some species can occur in many dry localities, including even arid places in deserts (KIREJTSHuK \& NeL, 2013). Adults of some species can be predators, living under the vegetation and feeding on microinvertebrates (KLAUSNITZER, 2009b), and a few species are associated with decaying animal matter (PEREIRA FARIAS et al., 2013). Regarding the Iranian fauna, KLAUSNITZER $(2016 \mathrm{~b}, \mathrm{c})$ listed 18 species of Scirtidae for Iran including two new species.

Iran occupies a large part of the Iranian plateau, and covers an area of 1,623,779 $\mathrm{km}^{2}$. Climatologically, Iran is a predominantly arid and semi-arid country, but the northern slopes of the Alburz ranges and the Caspian lowland receive 800 to $2000 \mathrm{~mm}$ annual rainfall, making them the most humid parts of the country. The Dasht-e Kavir and Dasht-e 
Lut deserts are the driest areas with an annual precipitation of less than $150 \mathrm{~mm}$. The highlands receive between 250 and $800 \mathrm{~mm}$ (ZEHZAD et al., 2002).

\section{MATERIAL AND METHODS}

The published data on distribution of the families Clambidae, Eucinetidae and Scirtidae (Coleoptera: Scirtoidea) in Iran are summarized by province. Genera and species are listed alphabetically. The following data are included in the catalogue for each species: (1) valid name, (2) junior synonym(s) used in literature about Iran, (3) published Iranian records arranged by province (classified by alphabetical order of Iranian province names) and the relevant references (classified by chronological order), (4) synoptic information on general distribution on a world scale and feeding habits. Data about classification, nomenclature and distribution are according to LÖBL \& LÖBL (2016 for Clambidae and Eucinetidae) and KlaUSNITZER (2016a for Scirtidae); in other situations, the related references are given. When accurate data about local distribution in Iran are lacking in a quoted reference, the mention "Iran (no locality cited)" is used. Additionally, the name "Persia" (as well as its equivalent German name "Persien") has been retained because this is traditional synonym for Iran. The provinces of Iran are represented in figure 1 .

\section{RESULTS}

Totally 27 species of Scirtoidea (Clambidae: 3 species from one genus, Eucinetidae: one species, Scirtidae: 23 species from 5 genera) are represented in the Iranian fauna. Clambus pilosellus Reitter, 1876 (Clambidae) and Contacyphon buceros Nyholm, 1949 (Scirtidae) are new records for the fauna of Iran. The list of species is given below with distribution data.

Series Scirtiformia Fleming, 1821

Superfamily Scirtoidea Fleming, 1821

Family Clambidae Fischer von Waldheim, 1821

Subfamily Clambinae Fischer von Waldheim, 1821

Genus Clambus Fischer von Waldheim, 1821

Clambus caucasus Endrödy-Younga, 1960

Distribution in Iran: Qazvin (SAKENIN et al., 2018).

General distribution: Azerbaijan, Caucasus, Italy, Turkey.

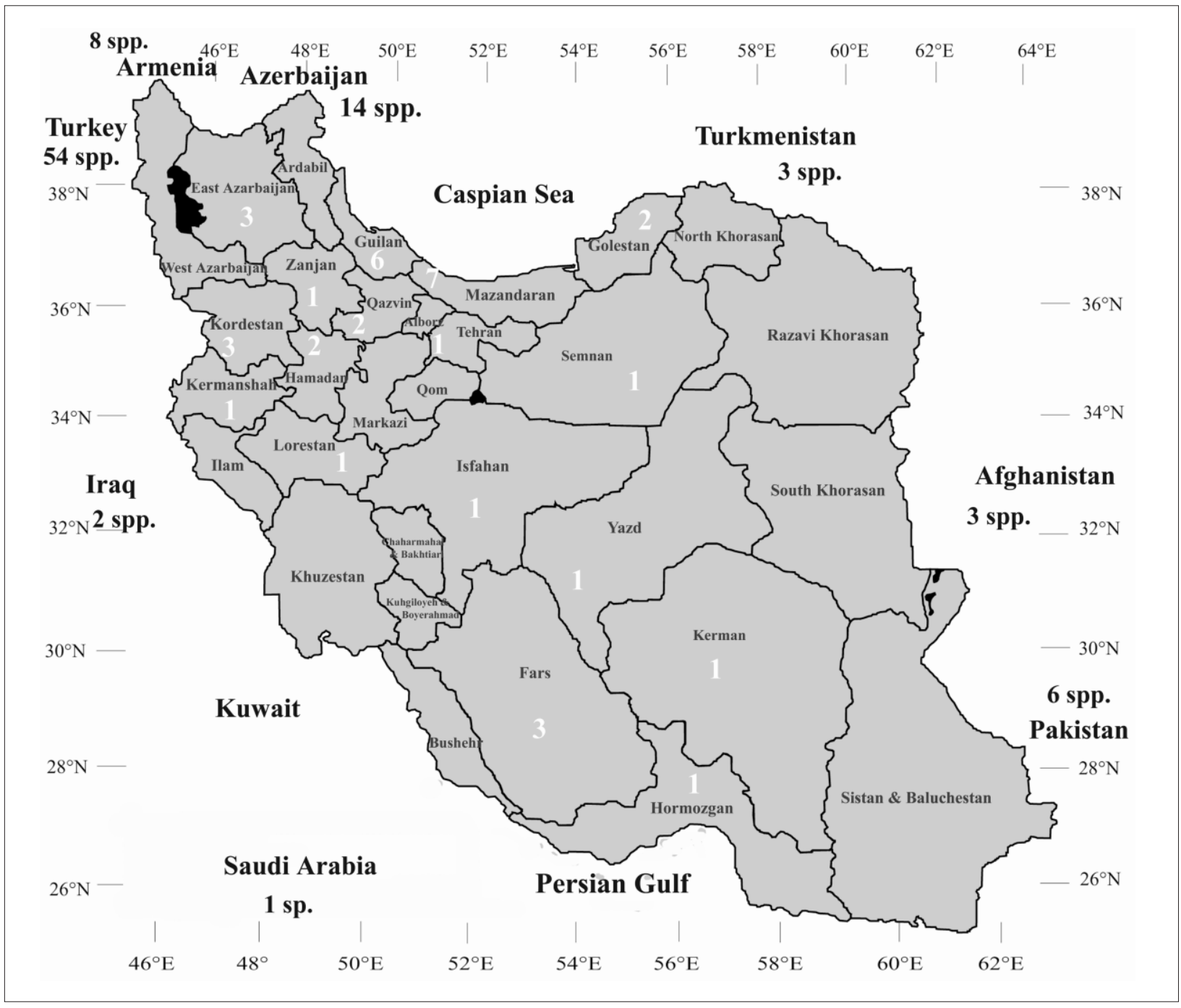

Fig. I - Map of Iran with provincial boundaries. 
Clambus dux xerxes Endrödy-Younga, 1960

Distribution in Iran: "Persien" (Endrödy-Younga 1960), Iran (no locality cited) (KLAUSNITZER \& MERK,L 1987; LÖBL \& LöBL, 2016).

General distribution: Caucasus, Iran, Israel, Pakistan.

Clambus pilosellus Reitter, 1876 (Fig. II)

Material examined. East Azarbaijan province, Azarshahr, $20 \mathrm{~km}$ E Nadiloo, 3743'N 4554'E, $117 \mathrm{~m}, 1 \mathrm{ex}$, 10.viii.2004. New record for Iran.

General distribution: Austria, Azerbaijan, Bulgaria, Croatia, Georgia, Hungary, Italy, Nepal, Romania, Slovakia, Taiwan, Turkey.

Family Eucinetidae Lacordaire, 1857 Genus Bisaya Reitter, 1884

\section{Bisaya nossidiiformis Reitter, 1884}

Distribution in Iran: Iran (no locality cited) (LÖBL \& LÖBL 2016).

General distribution: Azerbaijan, Iran.

Family Scirtidae Fleming, 1821

Genus Contacyphon Gozis, 1886

Comments: All the below species have been recorded from Iran under Cyphon spp.

Contacyphon algenib (Klausnitzer, 2003)

Synonym: Cyphon simulans Klausnitzer, 1990: 160.

Distribution in Iran: Guilan (Klausnitzer 1990 as $C$. simulans; KLAUSNITZER 2003), Mazandaran (KlauSNITZER, 2012b).

General distribution: Endemic to Iran.
Contacyphon arietizans (Nyholm, 1970)

Distribution in Iran: Iran (no locality cited) (KLAusNITZER, 2009b).

General distribution: Armenia, Georgia, Iran, Turkey.

Contacyphon buceros (Nyholm, 1949) (Fig. II)

Material examined: Guilan province, Astara (in bogs of

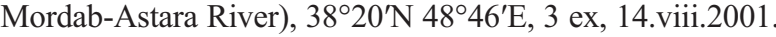
New record for Iran.

General distribution: Armenia, Azerbaijan, Georgia, Russia (South European Territory), Turkey.

Contacyphon caspicus (Klausnitzer, 1975)

Distribution in Iran: Iran (no locality cited) (KLAUSNITZER, 2006).

General distribution: Azerbaijan (KLAusnitzer, 1975a, 1976), Iran (KLAUSNITZER, 2006).

Comments: Occurrence of C. caspicus in Iran is likely, although it has not been recorded so far from any precise locality.

Contacyphon laevipennis (Tournier, 1868)

Synonym: Cyphon phragmiteticola Nyholm, 1955.

Distribution in Iran: East Azarbaijan, Mazandaran (Klausnitzer, 1990 as C. phragmiteticola; KLAUSNITZER, 2012b), Guilan (Klausnitzer, 1981 as C. phragmiteticola; KLAUSNITZER, 2012b), Iran (no locality cited) (KLAUSNITZER, 2009b).

General distribution: Afghanistan, Albania, Armenia, Austria, Azerbaijan, Bulgaria, Byelorussia, Czech Republic, Denmark, Egypt, Estonia, Finland, France, Germany, Great Britain, Greece, Hungary, Iran, Iraq, Israel, Italy, Kazakhstan, Latvia, Lithuania, Luxembourg, Moldavia, Mongolia, Morocco, Netherlands, North Korea, Norway, Poland, Russia (Central European Territory, East Siberia,

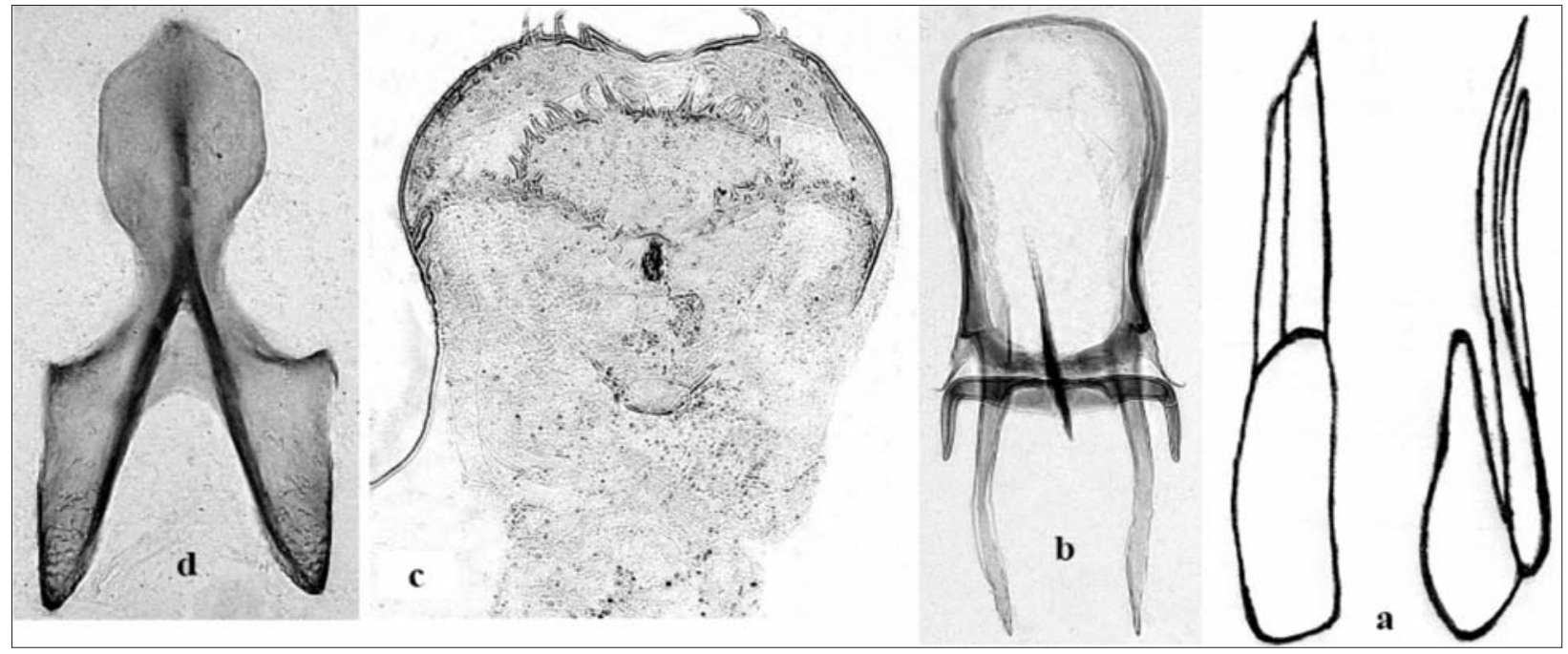

Fig. II - a: Clambus pilosellus Reitter, 1876: Penis (lateral and dorsal view). b-d: Contacyphon buceros Nyholm, 1949 (b: Penis, c: Prehensor, d: Tegmen). 
North European Territory, South European Territory), Saudi Arabia, Slovakia, Spain, Sweden, Switzerland, Syria, Tadzhikistan, Tunisia, Turkey, Turkmenistan, Ukraine.

Contacyphon lepidulus (Nyholm, 1968)

Distribution in Iran: Guilan, Kordestan (KLAUSNITZER, 1990, 2012b), Iran (no locality cited) (KLAUSNITZER, 2009b; ZWICK et al., 2013).

General distribution: Azerbaijan, Cyprus, Greece, Iran, Iraq, Syria, Turkey.

Contacyphon longiusculus (Nyholm, 1970)

Distribution in Iran: Mazandaran (KLAUSNITZER, 2012b), Iran (no locality cited) (KLAUSNITZER, 2009b).

General distribution: Azerbaijan, Georgia, Greece, Iran, Israel, Turkey.

Contacyphon ochraceus ochraceus (Stephens, 1830)

Distribution in Iran: Iran (no locality cited) (SHAVRIN et al., 2015).

General distribution: Austria, Byelorussia, Czech Republic, Denmark, Finland, France, Germany, Great Britain, Hungary, Iran, Ireland, Italy, Kazakhstan, Latvia, Lichtenstein, Luxembourg, Netherlands, Norway, Poland, Russia (Central European Territory, North European Territory, South European Territory), Slovakia, Spain, Sweden, Switzerland, Turkey, Ukraine.

Contacyphon palustris (C. G. Thomson, 1855)

Distribution in Iran: East Azarbaijan, Golestan, Kordestan (KLAUSNITZER, 1990, 2012b, c).

General distribution: Albania, Algeria, Austria, Azerbaijan, Bulgaria, Byelorussia, Cyprus, Czech Republic, Denmark, Finland, France, Germany, Great Britain, Greece, Hungary, Iran, Ireland, Israel, Italy, Kazakhstan, Latvia, Lebanon, Liechtenstein, Lithuania, Luxembourg, Netherlands, North Korea, Norway, Poland, Romania, Russia (Central European Territory, Far East, North European Territory, South European Territory), Slovakia, Spain, Sweden, Switzerland, Syria, Turkey, Ukraine.

Contacyphon pareuoplus (Klausnitzer, 2012)

Distribution in Iran: Mazandaran (KLAUSNITZER, 2012b), Iran (no locality cited) (ZwICK et al., 2013).

General distribution: Endemic to Iran.

Contacyphon perses (Nyholm, 1976)

Distribution in Iran: Guilan (Assalem - Holotype) (NYHOLM, 1976; KLAUSNITZER, 1990), Mazandaran (KLAUSNITZER, 1990, 2012b, c), Iran (no locality cited) (ZwICK et al., 2013).

General distribution: Endemic to Iran.

Contacyphon reitteri (Klausnitzer, 1976)

Distribution in Iran: Iran (no locality cited) (KLAUSNITZER, 2006).
General distribution: Azerbaijan (Lerik) (KLAUSNITZER, 1976), Iran (KLAUSNITZER, 2006).

Comments: Lerik is located near the Iranian border in southern Azerbaijan, between the tall mountains of the Talysh Mountains and the agricultural plain of the Lankaran Lowland.

Contacyphon simplex (Klausnitzer, 1981)

Distribution in Iran: Hamadan, Kermanshah, Kordestan, Mazandaran (Klausnitzer, 1990, 2012b, c), Tehran (Emameh - Holotype) (KlAUSNITZER, 1981), Iran (no locality cited) (ZwICK et al., 2013).

General distribution: Endemic to Iran.

Contacyphon sp.

Distribution in Iran: Fars (OSTOVAN \& NIAKAN, 2005, 2006).

Genus Elodes Latreille, 1797

Elodes eberti Klausnitzer, 1970

Distribution in Iran: Fars, Zanjan (KLAUSNITZER, 1970, 1972, 2009b), Tehran (KLAUSNITZER, 1990, 2000, 2009b, 2012b), Iran (no locality cited) (KLAUSNITZER, 2001, 2009a, 2012a, 2016a).

General distribution: Azerbaijan, Iran, Turkey, Ukraine.

Elodes frenzeli Klausnitzer, 2016

Distribution in Iran: Isfahan (Quamsar, $10 \mathrm{~km} \mathrm{SW,}$ Kamoo) (KLAUSNITZER, 2016c).

General distribution: Endemic to Iran.

Elodes orientalis Iablokoff-Khnzorian, 1973

Distribution in Iran: Hamadan (KLAUSNITZER, 2012b, c).

General distribution: Iran, Tadzhikistan.

Elodes persicus Klausnitzer, 1975

Distribution in Iran: Golestan (Gole Lovae) (KLAUSNITZER, 1975b, 2000, 2009a, 2012a, b), Semnan (KLAUSNITZER, 2012b, c), Iran (no locality cited) (KLAUSNITZER, 2001, 2016a).

General distribution: Endemic to Iran.

Elodes unicolor Klausnitzer, 2012

Distribution in Iran: Kerman, Yazd (KLAUSNITZER, 2012b).

General distribution: Endemic to Iran.

Elodes sp.

Distribution in Iran: Qazvin (Klausnitzer 1990 as Helodes sp.).

Genus Hydrocyphon Redtenbacher, 1858

Hydrocyphon consolatorius Klausnitzer, 1990

Distribution in Iran: Fars (KLAUSNITZER, 1990, 2012b), Iran (no locality cited) (Yoshitomi \& KlaUSNITZER, 2003; Yoshitomi, 2012; KLAUSNITZER, 2016a).

General distribution: Endemic to Iran. 
Hydrocyphon keili Klausnitzer, 2016

Distribution in Iran: Hormozgan (Sikhoran) (KLAUSNITZER, 2016b).

General distribution: Endemic to Iran.

Hydrocyphon segrex Nyholm, 1972

Distribution in Iran: Lorestan (KLAUSNITZER, 1981, 2009b, 2012b), Iran (no locality cited) (YoSHITOMI \& KLAUSNITZER, 2003; Yoshitomi, 2012; KLAUSNITZER, 2016a).

General distribution: Caspian Sea (Yoshiтomi, 2012), Greece, Azerbaijan, Iran, Turkey (Yoshitomi \& KLAusNITZER, 2003; KLAUSNITZER, 2016a).

Genus Microcara C. G. Thomson, 1859

Microcara luteicornis Reitter, 1888

Distribution in Iran: Mazandaran (KLAUSNITZER, 2012b, c).

General distribution: Azerbaijan, Georgia, Iran, Russia (South European Territory), Turkey.

\section{Genus Scirtes Illiger, 1807}

Scirtes orbicularis Panzer, 1793

Distribution in Iran: Guilan (KLAUSNITZER, 2012b, c).

General distribution: Austria, Azerbaijan, Belgium, Byelorussia, Czech Republic, Denmark, France, Georgia, Germany, Great Britain, Hungary, Ireland, Italy, Latvia, Netherlands, Poland, Russia (Central European Territory, South European Territory), Slovakia, Sweden, Switzerland, Turkey, Ukraine.

\section{DISCUSSION}

This checklist comprises 27 species within seven genera among which the genus Contacyphon with 13 recorded species is the most species-rich in Iran. Thus, of the 410 recorded species within 29 genera of Scirtoidea in the Palaearctic Region (LÖBL \& LÖBL 2016; KLAUSNITZER, 2016a), just 6.6\% have so far been recorded from Iran. Also, nine species are endemic to Iran. Iran comprises 31 provinces and Scirtoidea have been collected from just 17 of them. The highest diversity is found in the provinces Mazandaran and Guilan with seven and six species, respectively (Fig. I). No species are known from other 14 provinces lack of sampling. The exact localities of six species are unknown (Iran - no locality cited). The checklist indicates that most species have been recorded from northern parts of Iran (Guilan and Mazandaran provinces), comprising various aquatic ecosystems. Additionally, samplings on Iranian Scirtoidea, allowing a probably better knowledge of the regional fauna, have not been so far organized systematically. Among the neighbouring countries of Iran, the fauna of Turkey, with 54 recorded species has been studied better than others, followed by Russia (Central European Territory, East Siberia, Far East, North European Territory, South European Territory, West Siberia) and Azerbaijan with 49 and 14 species, respectively (LöBL \& LöBL, 2016; KLAUSNITZER, 2016a) (Fig. I). Additionally, Turkey and Azerbaijan both with 14 species, share the highest number of species with Iran than any other countries, followed by Russia (6), Armenia (3), Iraq (2), Afghanistan (1), Pakistan (1), Saudi Arabia (1), and Turkmenistan (1). However, since Iran is a large country with highly heterogeneous geographical regions and climates, surely there remain many further species to be discovered. Upon the several samplings and the fauna of areas adjacent Iran and other Palaearctic regions, this list is far from being complete. It is likely that the actual number of species of Scirtoidea occurring in Iran would probably exceed 50 species; further faunistic surveys systematically organized in several provinces of the country are strongly needed, as well as a more complete study of the available unidentified material deposited in different museums.

\section{ACKNOWLEDGEMENTS}

The authors are grateful to Dr. Rafal Ruta (University of Wroclaw, Poland), Dr. Petr Kment (National Museum of Prague, Czech Republic), Dr. Kee-Jeong Ahn and Dr. Jeong-Hun Song (Chungnam National University, South Korea), Dr. Csaba Csuzdi (Eszterházy Károly University, Hungary), and Dr. Peter Zwick (Schlitz, Germany) for contributions to this project. The research was supported by Islamic Azad University (Yadegare- Imam Khomeini (RAH) Shahre Rey Branch) and Senckenberg Deutsches Entomologisches Institut.

\section{REFERENCES}

EndRÖDY-YoungA S., 1960 - Monographie der Palaearktischen Arten der Gattung Clambus (Col., Clambidae). - Acta Zoologica Academiae Scientiarum Hungaricae, 6: 257-303.

GuÉORGUIEV B., 2004 - Eucinetidae - a new family to the fauna of Bulgaria (Coleoptera: Scirtoidea). - Historia naturalis bulgarica, 16: 113-117.

IABLOKOFF-KhnZORJAn S.M., 1973 - [Eine neue Art der Gattung Helodes Latreille aus Tadshikistan (Coleoptera, Helodidae)]. - Dokladi Akademii Nauk Armenskoj SSR 57, 112-114. [in Russian]

Kirejtshuk A.G., Nel, A., 2013 - New beetles of the suborder Polyphaga from the Lowermost Eocene French amber (Insecta: Coleoptera). - Annales de la Société entomologique de France (N.S.), 44 (4): 419-442.

KLAUSNITZER B., 1970 - Zur Kenntnis der Gattung Helodes Latr. (Col., Helodidae). - Entomologische Nachrichten, 14 (12): 177-184.

KLAUSNITZER B., 1972 - Zur Kenntnis der Gattung Helodes Latr. (Col., Helodidae). 2. Fortsetzung. - Entomologische Nachrichten, 16(4): 29-33.

Klausnitzer B., 1975a - Eine neue Art der Gattung Cyphon Paykull aus dem Gebiet des Kaspischen Meeres (Col., Helodidae). - Entomologische Nachrichten, 19: 6568.

Klausnitzer B., 1975b - Zwei neue Arten der Gattung Helodes Latreille aus der Palaearktis (Coleoptera, Helodidae). - Beiträge zur Entomologie, 25: 329-333.

Klausnitzer B., 1976 - Neue Arten, taxonomische und faunistische Bemerkungen zur europäischen CyphonFauna (Coleoptera, Helodidae). - Acta Entomologica Bohemoslovaca, 73: 256-262.

Klausnitzer B., 1981 - Zur Kenntnis der Cyphon- und Hydrocyphon-Fauna des Iran. Faunistische Abhandlungen. - Staatliches Museum für Tierkunde in Dresden, 8: 63-65.

KLAUSNITZER B., 1990 - Anmerkungen zur Helodidenfauna des Iran (Col.). - Entomologische Nachrichten und Berichte, 34(4): 159-165.

Klausnitzer B., 2000 - Eine neue Art der Gattung Elodes Latreille, 1796 aus der Kaukasus-region (Coleoptera, Scirtidae). - Entomologische Blätter, 96: 24-30. 
KLAUSNItZer B., 2001 - Eine neue Art der Gattung Elodes Latreille, 1796 aus Marokko (Col., Scirtidae). Entomologische Nachrichten und Berichte, 45: 2001/2, 123-126.

KLAUSNITZER B., 2003 - [new name]. In: Klausnitzer B. \& Yoshitomi H.: Notes on some homonyms and synonyms of the scirtid species (Coleoptera, Scirtidae). - Elytra, 31: 93-95.

Klausnitzer B., 2006 - Family Scirtidae Fleming, 1821. In: Löbl I., Smetana A. (eds.), Catalogue of Palaearctic Coleoptera. Volume 3. - Apollo Books, Stenstrup, pp. 316-323.

KLAUSNITZER B., 2009a - Contribution to the knowledge of Elodes sericea species-group with description of two new species from Middle Asia (Coleoptera, Scirtidae). - Acta Entomologica Musei Nationalis Pragae, 49(2): 711-728.

Klausnitzer B., 2009b - Insecta: Coleoptera: Scirtidae. In: Süßwasserfauna von Mitteleuropa. Begründet von A. Brauer, herausgegeben von P. ZWICK. Band 20/17. Spektrum Akademischer Verlag, Heidelberg. 326 S., 1041 Abb., 8 Farbtafeln.

KlaUsNitzer B., 2012a - A new species of the genus Elodes Latreille from Pakistan (Coleoptera: Scirtidae). Stuttgarter Beiträge zur Naturkunde A, Neue Serie, 5: 191-194.

Klausnitzer B., 2012b - Zur Kenntnis der Scirtidae des Iran mit Beschreibung je einer neuen Art aus den Gattungen Cyphon Paykull, 1799 und Elodes Latreille, 1796. - Beiträge zur Entomologie, 62(2): 361-374.

Klausnitzer B., 2012c - Neufunde von Scirtidae (Coleoptera) aus der Westpaläarktis und Ergänzungen zum "Catalogue of Palaearctic Coleoptera. Volume 3 (Scirtidae)" III. - Entomologische Nachrichten und Berichte, 56(2): 147-154.

KLAUSNITZER B., 2016a - Family Scirtidae. In: Löbl I., Löbl D. (ed.), Catalogue of Palaearctic Coleoptera, vol. 3, Scarabaeoidea, Scirtoidea, Dascilloidea, Buprestoidea, Byrrhoidea. Second revised and updated edition. Brill, pp. 412-425.

Klausnitzer B., 2016b - Eine neue Art der Gattung Hydrocyphon L. Redtenbacher, 1858 und Anmerkungen zur Fauna des Iran (Coleoptera, Scirtidae). Entomologische Nachrichten und Berichte, 60(1): 37-43.

Klausnitzer B., 2016c - Eine neue Art der Elodes sericeaArtengruppe (Coleoptera, Scirtidae) aus dem Iran. Entomologische Nachrichten und Berichte, 60(3/4): 205211.

Klausnitzer B., 2017 - Icones insectorum Europae centralis. Coleoptera: Scirtidae. - Folia Heyrovskyana, series B, 29: 1-17.

KlausnitZer B., MerkL O., 1987 - Helodidae, Eucinetidae and Clambidae of the Kiskunság National Park (Coleoptera: Dascilloidea). - The fauna of the Kiskunság National Park, 187, pp. 180-181.

Klausnitzer B., PosPisil P., 1991 - Larvae of Cyphon $s p$. (Coleoptera, Helodidae) in Ground Water. - Aquatic Insects, 13(3): 161-165.

KRYZHANOVSKIJ O.L., 1989 - Key of suborders and families. In: Ler P.A. (ed.), Opredelitel nasekomych Dalnego Vostoka SSSR, 3(1): 44-66. [in Russian]

LAWRENCE J.F., 1991 - Clambidae (Scirtoidea) (including Calyptomeridae). In: Stehr F.W. (ed.), Immature Insects, vol. 2. - Dubuque: Kendall-Hunt, pp. 365-366.

LAWRENCE J.F., NEWTON A.F., 1980 - Coleoptera associated with the fruiting bodies of slime moulds (Myxomycetes). The Coleopterists Bulletin, 34: 129-143.

LaWrence J.F., Newton A.F., 1995 - Families and subfamilies of Coleoptera (with selected genera, notes, references and data on family-group names). In: Pakaluk, J., Slipiński, S.A. (eds), Biology, phylogeny, and classification of Coleoptera: papers celebrating the $80^{\text {th }}$ birthday of Roy A. Crowson. Muzeum i Instytut Zoologii PAN, Warszawa: 779-1006 + $48 \mathrm{pp}$.

LESCHEN R.A.B., 2016 - 10.2. Eucinetidae Lacordaire, 1857. 10.3. Clambidae Fischer, 1821. In: Beutel R.G., Leschen R.A.B. (eds), Handbook of zoology, Vol. IV Arthropoda: Insecta. Part 38, $2^{\text {nd }}$ edition. Coleoptera, Vol. 1: Morphology and Systematics (Archostemata, Adephaga, Myxophaga, Polyphaga (partim). - Berlin, New York: Walter De Gruyter

LöBL I., LöBL D., 2016 - Family Eucinetidae, pp. 425-426; family Clambidae, p. 426. In: Löbl I., Löbl D. (ed.), Catalogue of Palaearctic Coleoptera, vol. 3, Scarabaeoidea, Scirtoidea, Dascilloidea, Buprestoidea, Byrrhoidea. Second revised and updated edition. - Brill, $983 \mathrm{pp}$.

Majka C.G., LANGOR, D.W., 2009 - Clambidae (Coleoptera) of Atlantic Canada. - Journal of the Acadian Entomological Society, 5: 32-40.

MAJKA C.G., 2010 - Eucinetidae (Coleoptera) of the Maritime provinces of Canada. - Journal of the Acadian Entomological Society, 6: 16-21.

Marinoni R.C., Ganho, N.G., Monné, M.L., Mermudes, J.R.M., 2001 - Hábitos Alimentares em Coleoptera (Insecta). - Ribeirão Preto, Holos Editora, 63 pp.

Mirsud D., 2001 - First record of Eucinetidae (Coleoptera) from the Maltese Islands. - The Central Mediterranean Naturalist, 3(3): 101-102.

NIKITSKY N.B., 1989 - 31. Eucinetidae. In: Ler P.A. (ed.), Opredelitel nasekomych Dalnego Vostoka SSSR, 3(1): 436-438. [in Russian]

Nyнolm T., 1968 - Neue paläarktische Arten der Gattung Cyphon PAYK. IV. Studien über die Familie Helodidae. IX. - Entomologisk Tidskrift, 89: 250-254.

Nyнolm T., 1970 - Beiträge zur Kenntnis der paläarktischen Helodiden. 1. Cyphon-Arten aus der Türkei. Studien über die Familie Helodidae. XI. Entomologisk Tidskrift, 91: 24-41.

Nyнolm T., 1972 - Drei neue Hydrocyphon-Arten aus dem Mittelmeergebiet (Coleoptera). - Entomologica scandinavica, 3: 33-39.

Nyнolm T., 1976 - Neue paläarktische Arten der Gattung Cyphon Paykull. V. (Col., Helodidae). Studien über die Familie Helodidae. XVII. - Entomologica Scandinavica, 7: 283-292.

Ostovan H., Niakan J., 2005 - Faunistic and biocenotic studies on aquatic beetles in southern and northern shores of Parishan Lake. - Journal of Agricultural Sciences (Islamic Azad University), 10(4): 93-116.

Ostovan H., Niakan J., 2006 - Faunistic and biocenotic studies on aquatic beetles in eastern and western shores of Parishan Lake. - Journal of Agricultural Sciences (Islamic Azad University), 11(3): 5-18.

Pereira Farias R.C.A., Dal-Bó D., Dos Santos W.E., CREÃo-Duarte A.J., Thyssen P.J., 2013 - First record of Scirtidae (Coleoptera) associated with decaying carcasses in the Neotropical Region. - EntomoBrasilis, 6(2): $171-172$.

Sakenin H., Samin N., Háva J., Bunalski M., Naderian H., KuBisz D., 2018 - A contribution to the study of Iranian Coleoptera with some new records. - Wiadomości Entomologiczne, 37(2): 96-109.

Shavrin A.V., ANichtchenKo A.V., BARSEVski A., 2015 Contribution to the knowledge of myrmecophilous beetles (Insecta, Coleoptera) of Latvia. - Linzer biologische Beiträge, 47/2: 1829-1842. 
VIT S., 1999 - Sur les genres Nycteus Latreille, 1829, et Eucinetus Germar, 1818 (Coleoptera, Eucinetidae). Mitteilungen der Schweizischen Entomologischen Gesellschaft, 72(3-4): 387-394.

VIT S., 2000 - Contribution à la connaissance de la famille Eucinetidae (Coleoptera). - Revue suisse de Zoologie, 107(1): 123-138.

Webster, R.P., SweEney J.D.; DeMerchant I., 2012 - New Coleoptera records from New Brunswick, Canada: Eucinetidae and Scirtidae. - ZooKeys, 179: 41-53.

Wheeler Q.D., Hoebeke E.R., 1984 - A review of mycophagy in the Eucinetoidea (Coleoptera), with notes on an association of the eucinetid beetle, Eucinetus oviformis, with a Coniophoraceae fungus (Basidiomycetes: Aphyllophorales). - Proceedings of the Entomological Society of Washington, 86: 274-277.

Yoshiтomi H., 2012 - Oriental Hydrocyphon (Coleoptera: Scirtidae: Scirtinae): Seven new species from Indonesia, Thailand, Malaysia, and India. - Psyche, 2012, 1-16.

Yoshitomi H., KLausnitzer B., 2003 - Scirtidae: World checklist of Hydrocyphon Redtenbacger, and revision of the Chinese species (Coleoptera). - Water beetles of China, Vol. III, pp. 519-537.
Young D.K., 2002a - Family 35. Eucinetidae Lacordaire 1857. In: Arnett, R.H., Thomas M.C., Skelley P.E., Frank J.H. (eds), American beetles. Volume 2. Polyphaga: Scarabaeoidea through Curculionidea. - CRC Press, Boca Raton, Florida, pp. 82-84.

Young D.K., 2002b - Family 36. Clambidae Jacquelin $d u$ Val 1857. In: Arnett R.H., Thomas M.C., Skelley P.E., Frank J.H. (eds), American beetles, Volume 2: Polyphaga: Scarabaeoidea through Curculionoidea. - CRC Press, Boca Raton, Florida, pp. 85-86.

Young D.K., 2002c - Family 37. Scirtidae Fleming 1821. In: Arnett R.H., Thomas M.C., Skelley P.E., Frank J.H. (eds), American beetles. Volume 2. Polyphaga: Scarabaeoidea through Curculionidea. - CRC Press, Boca Raton, Florida, pp. 87-89.

Zehzad B., Kiabi B.H., Madjnoonian H., 2002 - The natural areas and landscape of Iran: an overview. Zoology in the Middle East, 26(1): 7-10.

Zwick P., Klausnitzer B., Ruta R., 2013 - Contacyphon Gozis, 1886 removed from synonymy (Coleoptera: Scirtidae) to accommodate species so far combined with the invalid name, Cyphon Paykull, 1799). - Entomologische Blätter und Coleoptera, 109: 337-353. 
68 - Blank Page 